\title{
Qualifizierung in der Sozialen Arbeit
}

\author{
Soziale Arbeit studieren, praktizieren, beforschen und lehren
}

Qualifizierungsmöglichkeiten und -anforderungen in der Sozialen Arbeit sind fortwährenden Wandlungsprozessen unterworfen. Sie begründen sich sowohl in qualitativen wie quantitativen Personalbedarfen der Fachpraxis, in hochschul-, bildungs- und sozialpolitischen Entscheidungen als auch in professionspolitischen Bestrebungen der Sozialen Arbeit selbst. Diskussionen um die angemessene Form der Qualifizierung von Praktiker_innen der Sozialen Arbeit, von Lehrenden in entsprechenden Studiengängen wie auch von Forschenden, die vielfältige Probleme und Fragestellungen aus der Sozialen Arbeit untersuchen, sind keinesfalls neu. Das macht es jedoch nicht weniger relevant, aktuelle Entwicklungen, Diskussionsschwerpunkte und Herausforderungen in den Blick zu nehmen.

$\mathrm{D}$ ie bundesdeutsche hochschulische Qualifizierung für und in der Sozialen Arbeit war seit Ende der 1960er Jahre im Wesentlichen durch zwei Stränge charakterisiert (vgl. vertiefend bspw. Rauschenbach 2020; Gäs-Zeh und Klomann 2018): Zum einen durch die universitären Studiengänge der Erziehungswissenschaft(en) mit dem Schwerpunkt der Sozialpädagogik und zum anderen durch das - seit Ende der 1970er-Jahre mögliche - Studium der Sozialarbeit (und später auch der Sozialpädagogik) an Fachhochhochschulen. An den Universitäten entwickelte sich die

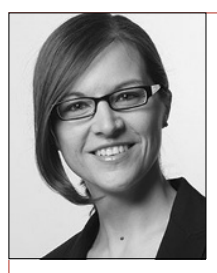

\section{Verena Klomann}

Abteilung Aachen,Katholische Hochschule NRW, Aachen, Deutschland

*1977, Prof. Dr. Dipl.-Sozialpädagogin (FH), M.A., Supervisorin (DGSv), Professorin für Theorien und Konzepte Sozialer Arbeit an der Katholischen Hochschule NRW

v.klomann@katho-nrw.de

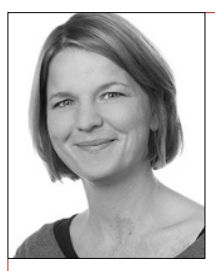

\section{Barbara Lochner}

Fakultät für angewandte Sozialwissenschaften,Fachhochschule Erfurt, Erfurt, Deutschland

*1978, Prof. Dr. Dipl.-Sozialpädagogin, M.A., Professorin für Pädagogik der Kindheit an der Fakultät für angewandte Sozialwissenschaften der Fachhochschule Erfurt

barbara.lochner@fh-erfurt.de

Zusammenfassung Der Beitrag führt in den Schwerpunkt ein, indem er aktuelle Entwicklungen in der Qualifizierungslandschaft der Sozialen Arbeit skizziert und disziplin- und professionspolitische Herausforderungen benennt.

Schlüsselwörter Qualifizierungswege Soziale Arbeit, Hochschule, Employability, Praxisbezug
Sozialpädagogik als spezifischer Teilbereich der Erziehungswissenschaft und - ausgehend von der wissenschaftlichen Disziplin - entstand eine Absolvent_innengruppe, die in der gesellschaftlichen Praxis ein Berufsfeld finden und eine „Professionalisierung von oben“ (Pfaffenberger und Schenk 1993, S. 228) vollziehen musste. Das Studium an Fachhochschulen war durch den Mangel an universitär ausgebildeten und promovierten Sozialarbeiter_innen wesentlich durch die sogenannten Bezugswissenschaften geprägt - für die Methodenlehre und die praxisbezogenen Inhalte waren lehrende Sozialarbeiter_innen zuständig (vgl. Schumacher 2011, S. 1). Thomas Rauschenbach beschreibt es so, dass diesem nahezu gleichberechtigten Nebeneinander von mehreren Fächern die „orientierende Mitte“ (Rauschenbach 1999, S. 153) fehlte. Dies brachte für die Studierenden nicht selten die Herausforderung mit sich, den spezifischen Auftrag sowie die spezifische Perspektive der Sozialen Arbeit eigenständig herauszuarbeiten und ein professionelles Bild von Sozialer Arbeit zu konstruieren (ebd.; Stock et al. 2016, S. 34 ff.). Von Seiten der Praxis wurde in dieser Zeit insbesondere der Verlust des Praxisbezugs kritisiert, der aus deren Sicht mit den wissenschaftlich ausgerichteten und zur kritischen Auseinandersetzung anregenden Studiengänge einherging (vgl. Gäs-Zeh und Klomann 2018, S. 159).

Ausgehend von den unterschiedlichen Entwicklungslinien waren die Studiengänge an den Universitäten eher wissenschaftlich-disziplinär und an den Fachhochschulen eher praxisorientiert geprägt. 1994 konstatierte Thole, dass die Soziale Arbeit "keinen eindeutigen, klar zu benennenden Ort in der Praxis, kein einheitliches Profil in der Ausbildung, keine selbstverständliche, von allen 


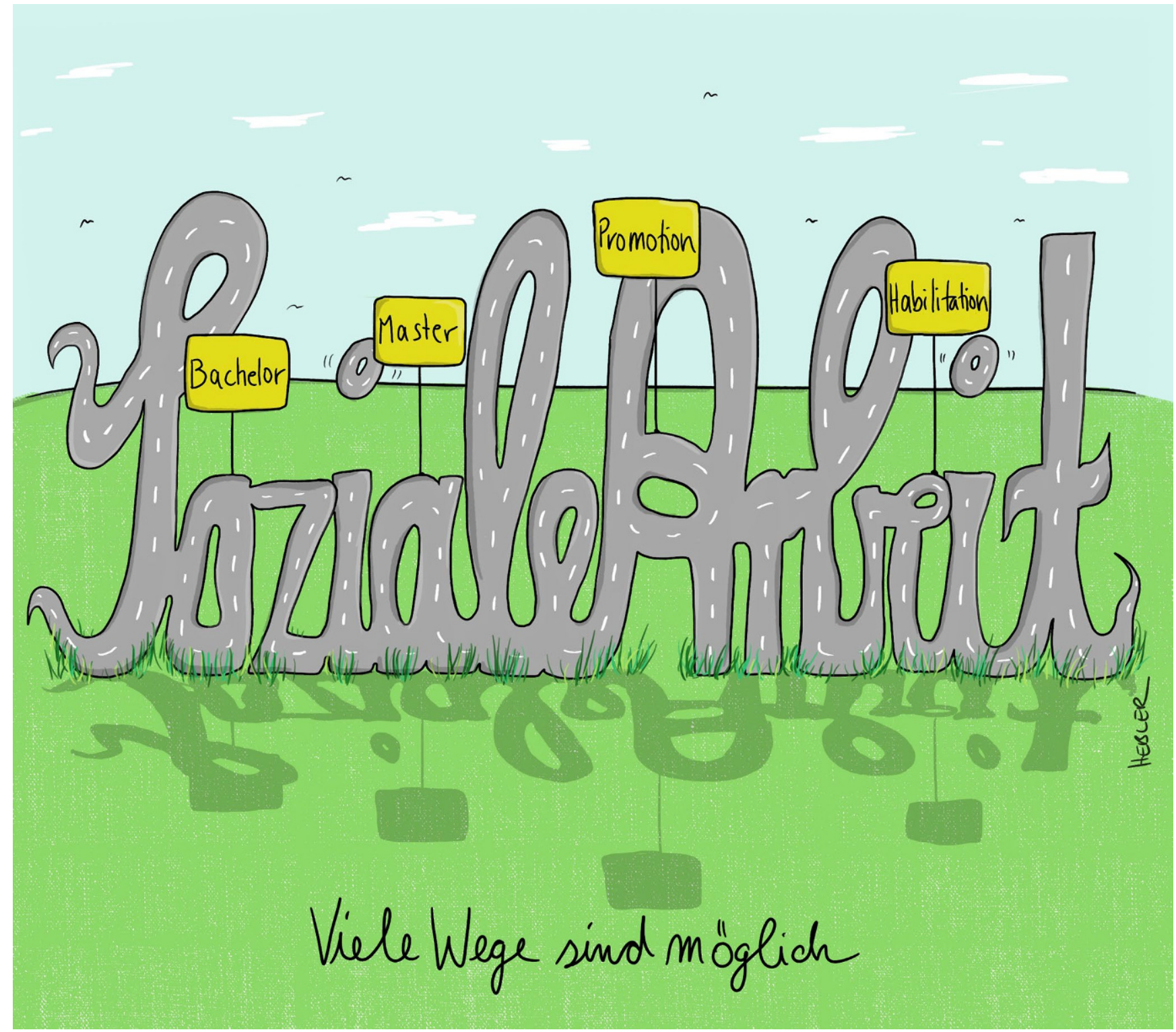

ihren VertreterInnen geteilte disziplinäre Heimat, keine stabilen theoretischen, wissenschaftlichen und professionellen Koordinaten und Referenzpunkte" habe (Thole 1994, S. 253 f.). Diese diffuse und herausfordernde Situation wurde durch den im Jahr 1999 beginnenden Bologna-Prozess und die hiermit verbundene und seit der Jahrtausendwende erfolgte gravierende Studienreform verschärft. Neben der einschneidenden Umstellung auf das Bachelor-Master-System rückte hiermit vor allem die sogenannten „Employability“ der Studienabsolvent_innen in den Mittelpunkt: Im Rahmen des Studiums soll also Beschäftigungsfähigkeit und damit Anschlussfähigkeit auf dem Arbeitsmarkt hergestellt werden. Folglich veränderte sich auch der Auftrag der Hochschulen: Sie sollen nun „zunehmend berufliche Kompetenzen vermitteln und somit eine Vorbereitung auf die berufliche Praxis ermöglichen “ (HRK 2013, S. 15). Neben dieser nachhaltigen Veränderung der Studienstrukturen und -ziele hat sich in den vergangenen 20 Jahren die Studienlandschaft in der Sozialen Arbeit erheblich ausdifferenziert: Neue Studiengänge wurden entwickelt und insbesondere duale Studienformate erleben einen großen Aufschwung. In den vergangenen zehn Jahren ist zudem - insbesondere vor dem Hintergrund eines anhaltenden und wachsenden, sowohl quantitativen als auch qualitativen Fachkräftebedarfes - eine steigende Anzahl an privaten Hochschulen zu verzeichnen, an denen das Studium der Sozialen Arbeit möglich ist (vgl. Klomann und Breuer-Nyhsen 2019, S. 12 f.). In Anbetracht dessen, dass hier nur diejenigen Studierenden können, die über die entsprechenden finanziellen Ressourcen verfügen, stellt sich die Frage, ob die Soziale Arbeit hier nicht eines ihrer zentralen Anliegen konterkariert: den Abbau von ungleichen Bildungschancen und -zugängen. 


\section{Extrablick: Ausbildung und Studium}

\section{Studierende der Sozialen Arbeit, Interessens- und Motivationslagen}

Grundsätzlich erfreulich ist das anhaltende Interesse und die anhaltende Nachfrage an Studiengängen und -plätzen der Sozialen Arbeit: Von den im Sommersemester 2018 mehr als 2,5 Mio. Studierenden an bundesdeutschen Hochschulen waren 83.224 Personen in der Fächergruppe Sozialwesen eingeschrieben, die die Studiengänge der Sozialen Arbeit, der Sozialpädagogik und es Sozialwesens umfasst. Das waren knapp zehn Prozent der Studierenden an Fachhochschulen insgesamt und knapp drei Prozent der Studierenden bundesweit (vgl. DESTATIS 2019). Die Studiengänge dieser Fächergruppe scheinen zudem insbesondere für sogenannte Bildungsaufsteiger_innen - also Studierende, die als Erste aus ihrer Familie eine Hochschule besuchen interessant zu sein: Im Wintersemester 2015/12016 kamen in den Studiengängen des Sozialwesens bundesweit $62 \%$ der Studierenden aus nicht-akademischen Haushalten (vgl. Bundesministerium für Bildung und Forschung 2017, S. 7). Die Studienentscheidung orientiere sich hier vor allem an der Frage, welche Studiengänge als gut beherrschbar und studierbar gelten (Mecheril und Klinger 2010, S. 96 f.), deren (auch finanzielles) Risiko einigermaßen überschaubar scheint und die einen zügigen und relativ sicheren Berufseinstieg ermöglichen. All dies ist bei den Studiengängen, die für die Soziale Arbeit qualifizieren, der Fall.

Das Studium dient zudem häufig primär der Erreichung des berufsqualifizierenden Abschlusses und nicht der Befähigung für eine wissenschaftliche Tätigkeit: Es wird oft als Zugangsvoraussetzung (und manchmal auch -hindernis) für die berufliche Tätigkeit verstanden (Kotthaus 2017, S. 108 ff.). Dies bestätigt auch das 12. Studierendensurvey: Bei Studierenden der Sozial- und Erziehungswissenschaften gilt das Studium dem „Erlangen einer Qualifikation und dem Erwerb von Employability, d. h. der Vorbereitung auf den Beruf zur Sicherung der eigenen Zukunft" (Bundesministerium für Bildung und Forschung 2014, S. 115 f.) - eine theoriegeleitete und methodische Ausbildung sowie neugieriges und forschendes Lernen sind eher nachrangig (vgl. ebd.; Bundesministerium für Bildung und Forschung 2017).

Insgesamt ist die Motivationslage der Studieninteressierten für Studiengänge, die für die Soziale Arbeit qualifizieren, vielfältig: Ein Studium ist - so wird es auch gesellschaftlich suggeriert - ,besser' als eine Ausbildung, Bachelor-Studiengänge (insbesondere an Fachhochschulen) sind klar strukturiert und oft schulähnlich organisiert und das Anforderungsprofil gilt als überschaubar. Als Motivatoren zählen auch die ausreichende Berufsqualifizierung durch den Bachelor-Abschluss sowie die anhaltende Nachfrage an entsprechendem Personal (vgl. Otto 2014, S. 751).

\section{Qualifizierung in der Sozialen Arbeit aktuell: Herausforderungen und Dilemmata}

An der o. g., eher ernüchternden Standortbestimmung von Werner Thole zum - in mehrfacher Hinsicht - fehlenden Fundament der bundesdeutschen akademischen Sozialpädagogik aus dem Jahr 1994 hat sich bis heute wenig geändert. Die unterschiedlichen Zugänge zur Weiterentwicklung der Qualifizierungswege in der Sozialen Arbeit sind mit heterogenen, mitunter kontroversen Intentionen verbunden (Küster und Schoneville 2012, S. 1062 f.). In ihrer Gemengelage etablierte sich ein von Diversität geprägtes, komplexes Ausbildungsund Wissenschaftssystem, das den unterschiedlichen Zielsetzungen und Interessen gerecht werden soll, Professionalitätsbestrebungen befördern, aber auch unterwandern kann (AGJ 2019, S. 10). So sind einerseits verschiedene Selbstbehauptungs- und Profilierungsbestrebungen in der Sozialen Arbeit zu erkennen, die aber andererseits nur selten konsensual von den universitären und (fach)hochschulischen Vertreter_innen der Sozialen Arbeit verfolgt werden. Hierzu gehören insbesondere die Weiterentwicklung von Promotionsmöglichkeiten in der Sozialen Arbeit, die Qualifizierung von Lehrenden für die entsprechenden Fachbereiche sowie die Auseinandersetzung damit, was grundsätzlich, aber insbesondere nach der Bologna-Reform als Nachweise fachlicher Kompetenz in der Sozialen Arbeit Geltung erlangt. Diskutiert werden in diesem Zusammenhang sowohl die Durchlässigkeit des Wissenschaftssystems für Absolvent_innen von Studiengängen der Sozialen Arbeit als auch die Anerkennung und Anschlussfähigkeit hochschulischer Abschlüsse in der Fachpraxis.

Dem Ziel, durch das Bachelor-Master-System die „Employability“ der Absolvent_innen zu erhöhen, scheint auf Seiten der Hochschulen dadurch begegnet worden zu sein, dass bei der Entwicklung neuer, lokaler Studiengänge „der Phantasie (...) kaum Grenzen gesetzt“ wurden (Rauschenbach 2020, S. 151), was es Anstellungsträgern erschwert, die mit einem Abschluss verbundenen Inhalte zu identifizieren. Zugleich haben sich in den vergangenen Jahrzehnten der Vorwurf der Praxisferne des Studiums und der geringen Praxistauglichkeit von Studienabsolvent_innen verfestigt. Dennoch hat sich auf der Seite der Fachpraxis der Bachelor als grundständige Qualifikation etabliert und es scheint bisher nur wenig Energie darauf verwandt worden zu sein, jenseits von Leitungsstellen in der öffentlichen Verwaltung Tätigkeitsprofile zu entwickeln, die auf einem Master-Abschluss aufbauen beziehungsweise diesen vo- 
raussetzen. Der Bachelor gilt als „Standardabschluss für die Berufseinmündung “ (Kruse 2017, S. 183; Schröder 2010, S. 138). Auf der anderen Seite werfen wachsende gesellschaftliche Ungleichheitsentwicklungen, zunehmende Krisensituationen und Problemfelder sowie komplexer werdende Anforderungen an die Soziale Arbeit die Frage auf, ob die erforderliche Qualifikation überhaupt im Rahmen eines (meist) sechssemestrigen Bachelorstudienganges erworben werden kann oder ob nicht vielmehr der Masterabschluss als Regelabschluss nötig wäre.

\section{Beiträge des Schwerpunkts}

In diesem Schwerpunkt greifen wir einige der skizzierten Entwicklungen sowie Perspektiven zur Qualifizierung in der Sozialen Arbeit auf und diskutieren diese unter verschiedenen Fragestellungen. Pandemiebedingt müssen wir dabei leider auf zwei vorgesehene Beiträge, die sich mit der Situation der Studierenden sowie der Zertifizierungsfrage in der Sozialen Arbeit beschäftigt hätten, verzichten.

- Andreas Polutta widmet sich in seinem Beitrag „Die Bedeutung von Praxis- und Theoriestudium für die Fachlichkeit Sozialer Arbeit" aktuellen Herausforderungen, Kontroversen und Perspektiven einer professionalitätsfördernden grundständigen Qualifizierung für die Soziale Arbeit. Hierbei greift er zentrale Entwicklungen in der Hochschullandschaft in Bezug auf die Soziale Arbeit sowie insbesondere die Debatten um duale Studiengänge auf und zeigt Konfliktlinien auf, die nicht nur für diese Studiengänge relevant sind.

- Monika Alisch und Michael May beleuchten in ihrem Beitrag „Möglichkeiten und Perspektiven der Promotion in der Sozialen Arbeit" die für die Soziale Arbeit spezifischen Promotionsbedingungen. Hierbei geht es auch um die Fragen, wie es gelingen kann, Absolvent innen von Studiengängen der Sozialen Arbeit für einen wissenschaftlichen Werdegang zu begeistern, wie Promotionen gefördert und begleitet werden können und wer die zukünftigen Lehrenden und Forschenden der Sozialen Arbeit sind.

- Diesen Aspekt greifen auch Anna-Lena Rademaker und Michael Stricker in ihrem Beitrag „Karrierewege FH-Professur" auf. Hierbei greifen Sie auf Erfahrungen aus der Modellphase dieses Projektes zur Förderung professoralen Nachwuchs in der Sozialen Arbeit an Fachhochschulen und Hochschulen für angewandte Wissenschaften in NRW zurück und identifizieren zentrale Bedarfe bei der Förderung von Karrierewegen in der Sozialen Arbeit.
Funding. Open Access funding provided by Projekt DEAL.

Open Access. Dieser Artikel wird unter der Creative Commons Namensnennung 4.0 International Lizenz veröffentlicht, welche die Nutzung, Vervielfältigung, Bearbeitung, Verbreitung und Wiedergabe in jeglichem Medium und Format erlaubt, sofern Sie den/die ursprünglichen Autor(en) und die Quelle ordnungsgemäß nennen, einen Link zur Creative Commons Lizenz beifügen und angeben, ob Änderungen vorgenommen wurden.

Die in diesem Artikel enthaltenen Bilder und sonstiges Drittmaterial unterliegen ebenfalls der genannten Creative Commons Lizenz, sofern sich aus der Abbildungslegende nichts anderes ergibt. Sofern das betreffende Material nicht unter der genannten Creative Commons Lizenz steht und die betreffende Handlung nicht nach gesetzlichen Vorschriften erlaubt ist, ist für die oben aufgeführten Weiterverwendungen des Materials die Einwilligung des jeweiligen Rechteinhabers einzuholen.

Weitere Details zur Lizenz entnehmen Sie bitte der Lizenzinformation auf http://creativecommons.org/licenses/by/4.0/deed.de

\section{Literatur}

Arbeitsgemeinschaft für Kinder- und Jugendhilfe (2019). Gesellschaftliche Anerkennung und Aufwertung der Sozialen Berufe in der Kinder- und Jugendhilfe - Fachkräfte gewinnen, Qualität erhalten und verbessern! Positionspapier der Arbeitsgemeinschaft für Kinder- und Jugendhilfe. https:// www.agj.de/fileadmin/files/positionen/2019/Aufwertung_Soziale_Berufe. pdf. Zugegriffen: 30. Juni 2020.

Bundesministerium für Bildung und Forschung (2014). Studiensituation und studentische Orientierungen. 12. Studierendensurvey an Universitäten und Fachhochschulen. Bonn/Berlin. https://www.bmbf.de/pub/Studierendensurvey_Ausgabe_11_Langfassung.pdf. Zugegriffen: 15. Okt. 2018.

Bundesministerium für Bildung und Forschung (2017). Studiensituation und studentische Orientierungen. 13. Studierendensurvey an Universitäten und Fachhochschulen. https://www.soziologie.uni-konstanz.de/typo3temp/ secure_downloads/101284/0/8f0c2b692bb060753bead044da8dfd2a4eb3 a54e/Studierendensurvey_Ausgabe_13_Hauptbericht.pdf. Zugegriffen: 28. Juni 2020.

DESTATIS (2019). Bildung und Kultur. Studierende an Hochschulen. Fachserie 11, Reihe 4.1, Sommersemester 2018. https://www.destatis.de/ DE/Themen/Gesellschaft-Umwelt/Bildung-Forschung-Kultur/Hochschulen/_inhalt.html. Zugegriffen: 1. Aug. 2019.

Gäs-Zeh, U., \& Klomann, V. (2018). Ausbildung und Studium der Sozialen Arbeit - Entwicklungslinien der beruflichen Sozialisation. In M. Gerards, U.-A. Lammel, N. Frieters-Reermann \& R. Krockauer (Hrsg.), Aachens Hochschule für Soziale Arbeit. 100 Jahre Tradition - ReflexionInnovation (S. 149-170). Opladen, Berlin, Toronto: Barbara Budrich.

Hochschulrektorenkonferenz (HRK) (2013). Employability und Praxisbezüge im wissenschaftlichen Studium. http://www.hrk-nexus.de/fileadmin/ redaktion/hrk-nexus/07-Downloads/07-02-Publikationen/Fachgutachten_ Employability.pdf. Zugegriffen: 8. Febr. 2017.

Klomann, V., \& Breuer-Nyhsen, J. (2019). Fachkräftebedarf und Hochschulausbildung - Eine kritische Diskussion von Interessenslagen und Entwicklungsperspektiven. Jugendhilfereport, 1(19), 11-17.

Kotthaus, J. (2017). Die Soziale Arbeit als Menschenrechtsprofession - revisited. In R. Braches-Chyrek \& H. Sünker (Hrsg.), Soziale Arbeit in gesellschaftlichen Konflikten und Kämpfen (S. 101-118). Wiesbaden: Springer VS.

Kruse, E. (2017). Aus- und Weiterbildung in der Sozialen Arbeit. In F. Kessl, E. Kruse, S. Stövesand \& WernerThole (Hrsg.), Soziale Arbeit Kernthemen und Problemfelder (S. 182-193). Opladen, Toronto: Budrich.

Küster, E.-U., \& Schoneville, H. (2012). Qualifizierung für die Soziale Arbeit. Auf der Suche nach Normalisierung, Anerkennung und dem Eigentlichen. In W. Thole (Hrsg.), Grundriss Soziale Arbeit (S. 1045-1066). Wiesbaden: Springer VS. 
Mecheril, P., \& Klinger, B. (2010). Universität als transgressive Lebensform. Anmerkungen, die gesellschaftliche Differenz und Ungleichheitsverhältnisse berücksichtigen. In L. Darowaska, T. Lüttenberg \& C. Machold (Hrsg.), Hochschule als transkultureller Raum? Kultur, Bildung und Differenz in der Universität (S. 83-116). Bielefeld: transcript.

Otto, H.-U. (2014). Akademisiert - aber nicht professionalisiert. Essay zur Sozialen Arbeit als personenbezogene Dienstleistung. In M. P. Schwarz, W. Ferchhoff \& R. Vollbrecht (Hrsg.), Professionalität: Wissen - Kontext. Sozialwissenschaftliche Analysen und pädagogische Reflexionen zur Struktur bildenden und beratenden Handelns (S. 750-755). Bad Heilbrunn: Klinkhardt.

Pfaffenberger, H., \& Schenk, M. (Hrsg.). (1998). Sozialarbeit zwischen Berufung und Beruf. Professionalisierungs- und Verwissenschaftlichungsprobleme der Sozialarbeit/Sozialpädagogik. Münster: Lit Verlag.

Rauschenbach, T. (1999). Sozialarbeitswissenschaft - eine neue Wissenschaft oder ein neues Leitbild? - Plädoyer für eine Versachlichung einer aufgeregten Debatte. Theorie und Praxis der Sozialen Arbeit, 50(4), 152158 .

Rauschenbach, T. (2020). Sozialpädagogik an drei Orten. Professionelle und disziplinäre Entwicklungen in den Hochschulausbildungen der Sozialen Arbeit. In P. Cloos, B. Lochner \& H. Schoneville (Hrsg.), Soziale Arbeit als Projekt (S. 145-158). Wiesbaden: Springer VS.

Schröder, J. (2010). Was sollen sie können? - Die aktuelle Fachdebatte über Kompetenzen von Fachkräften mit Bachelor- und Masterabschlüssen in der Kinder- und Jugendhilfe. Soziale Passagen, 2, 135-142. https:// doi.org/10.1007/s12592-010-0034-y.

Schumacher, T. (2011). Das Studium der Sozialen Arbeit als Puzzle. In T. Schumacher (Hrsg.), Die soziale Arbeit und ibre Bezugswissenschaften (S. 1-6). Stuttgart: Lucuis \& Lucuis.

Stock, C., Schermaier-Stöckl, B., Klomann, V., \& Vitr, A. (2016). Soziale Arbeit und Recht. Baden-Baden: Nomos. Lehrbuch

Thole, W. (1994). Sozialpädagogik an zwei Orten. Professionelle und disziplinäre Ambivalenzen eines noch unentschiedenen Projekts. In H.-H. Krüger \& T. Rauschenbach (Hrsg.), Erziehungswissenschaft. Die Disziplin am Beginn einer neuen Epoche (S. 253-274). Weinheim: Juventa.

Hier steht eine Anzeige. Springer 